\section{Was bestimmt die \\ Wirksamkeit eines Achtsamkeitstrainings}

Lindsay EK et al. Acceptance lowers stress reactivity: Dismantling mindfulness training in a randomized controlled trial. Psychoneuroendocrinology 2018; 87: 63-73

Mit achtsamkeitsbasierten Interventionen gelingt es die Stressreaktivität zu dämpfen. Allerdings ist wenig über die Mechanismen bekannt, die diese Wirkung bedingen. Amerikanische Wissenschaftler vermuteten, dass das Potential der Achtsamkeitsintervention von der Akzeptanz abhängt.

In eine dreiarmige Studie wurden 153 gestresste Erwachsene eingeschlossen. Die achtsamkeitsbasierten Komponenten wurden in drei 14-tägigen, Smartphonebasierten Ausbildungsprogrammen angeboten: Training in Achtsamkeit und Überwachung, Training in alleiniger Überwachung von Instruktionen sowie Training in analytischem Denken ohne Aufmerksamkeitsinstruktionen. Die Programme waren dabei in 15 Lektionen unterteilt. Die Studienteilnehmer wurden randomisiert jeweils einem Programm zugewiesen. Ausgewertet wurden die vollständigen Angaben von 144 Personen. Das Durchschnittsalter der Teilnehmer betrug 32 Jahre. Etwa 2 Drittel der Studienpopulation war weiblich. In der Gruppe Achtsamkeit und Überwachung (MA) wurden die Aufmerksamkeit im gegenwärtigen Moment und eine Orientierung der Akzeptanz trainiert. In der Gruppe alleiniger Überwachung von Instruktionen (MO) wurde trainiert, sich auf körperliche und emotionale Erfahrungen ohne Akzeptanzinstruktionen zu konzentrieren. In der dritten Gruppe wurde die Reflexion eigener Gedanken, Gefühle und Verhalten angeregt, die Teilnehmerinnen und Teilnehmer wurden aber nicht in Achtsamkeit unterwiesen. Hinsichtlich Alter, Geschlecht, Ausbildung, Ethnität und BMI unterschieden sich die Gruppenmitglieder nicht.

Die Stressreaktionen der Teilnehmer wurden mit dem Trier Social Stress Test (TSST) erfasst. Die Cortisolspiegel wurden aus Speichelproben zu 4 verschiedenen Zeitpunkten vor Studienbeginn und nach dem TSST gemessen. Darüber hinaus wurden systolischer und diastolischer Blutdruck in

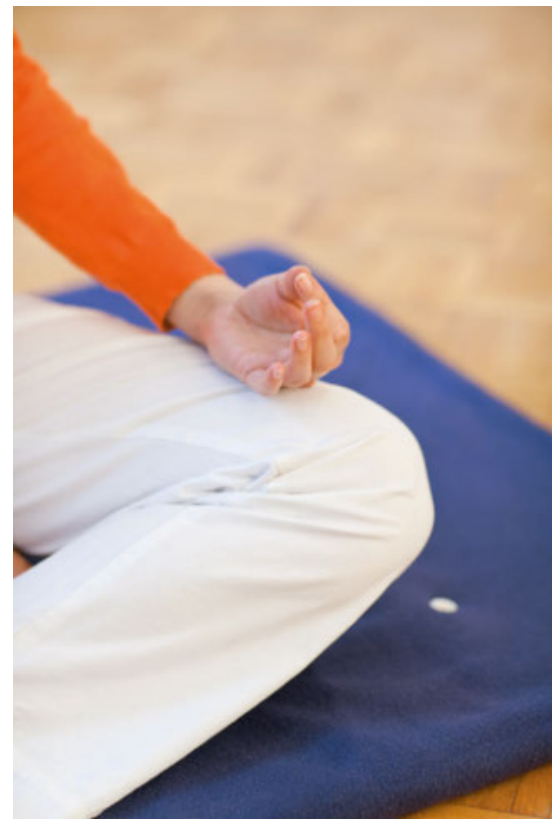

Ein kurz gefasstes achtsamkeitsbasiertes Fern-Training senkt wirksam die Cortisolund Blutdruckreaktivität. Das Erlernen der Akzeptanz ist entscheidend zur Effektsteuerung. (Quelle: Holger Münch / Thieme Verlagsgruppe)

2-minütigen Intervallen an 5 Zeitpunkten vor und im Verlauf der Untersuchung und nach dem TSST bestimmt.

Wie von dem Wissenschaftlerteam vermutet, reduzierte das Training von Aufmerksamkeit im gegenwärtigen Moment und Akzeptanz im Vergleich zu den beiden anderen Gruppen die Reaktivität von Cortisol und systolischem Blutdruck. Hinsichtlich der subjektiven Stressreaktivität ergaben sich allerdings keine Unterschiede zwischen den Gruppen.

\section{FAZIT}

Mit dieser Untersuchung konnte erstmals nachgewiesen werden, dass ein kurzgefasstes achtsamkeitsbasiertes Fern-Training wirksam die Cortisol- und Blutdruckreaktivität senkt und dass das Erlernen der Akzeptanz zur Effektsteuerung entscheidend ist, so die Autorinnen und Autoren.

Richard Kessing, Zeiskam 